ISSN: 1130-3743 - e-ISSN: 2386-5660

DOI: http://dx.doi.org/10.14201/teoredu2016281105129

\title{
PRIORIZACIÓN DE VALORES EN ESTUDIANTES JÓVENES Y MAYORES EN EL CONTEXTO DE LA CRISIS ECONÓMICA
}

\author{
Young and adult students prioritization of values \\ in the context of economic crisis
}

\section{Hiérarchisation des valeurs des étudiants jeunes et plus âgés dans le contexte de la crise économique}

\author{
María José Hernández Serrano, Gabriel Parra Nieto y \\ María Dolores PÉREZ GRANDE \\ Universidad de Salamanca. Facultad de Educación. Departamento de Teoría e \\ Historia de la Educación. Paseo de Canalejas, 169.37008 Salamanca.mjhs@ \\ usal.es; gabrielparra@usal.es; dpg@usal.es
}

Fecha de recepción: enero de 2016

Fecha de aceptación: marzo de 2016

\section{RESUMEN}

El cambio de valores en la sociedad postmoderna nos sitúa ante un debate recurrente sobre una posible brecha generacional entre jóvenes y mayores. La postmodernidad, el aumento del individualismo y el relativismo contrastan hoy con las necesidades que plantean la situación económica y la reformulación de las prioridades axiológicas. Ante las circunstancias actuales, cuestionamos si la crisis mundial no ha venido a homogeneizar en cierta medida los valores de jóvenes y mayores, al vivir ambos etapas de socialización en contextos de mayor escasez e inseguridad. El objetivo de este estudio es comparar las similitudes y diferencias en la apreciación de los valores de ambas cohortes, según la escala Rvs (Rokeach). En los resultados encontramos considerables semejanzas en los valores básicos que priorizan ambos grupos. Pero también se sigue evidenciando en los jóvenes un mayor índice de 
postmodernismo al presentar una mayor pluralidad y diversificación hacia valores más individualistas e intrapersonales.

Palabras clave: valores; postmodernidad; crisis económica; estudiantes universitarios; estudiantes mayores.

\section{SUMMARY}

Changing of values in the postmodern society rekindle the recurring debate about a possible generational gap between young and adult people. Postmodernism, the increasing individualism and relativism, as constants in advanced current societies contrast with the needs posed by the economic crisis and the reformulation of value priorities. Under this context, we question whether the global crisis is homogenizing the values prioritized by the two generations, since both may have been influenced by a context of increased scarcity and insecurity. The aim of this study is to compare the similarities and differences in the appreciation of the values of both cohorts, according to Rvs scale (Rokeach). Results found considerable similarities in the basic values that prioritize both cohorts. However, a higher rate of postmodernism is also evidenced in the young cohort, as they showed a greater values plurality and diversification towards more individualistic and intrapersonal values.

Key words: values; postmodernism; economic crisis; college students; adult students.

\section{SOMMAIRE}

La modification des valeurs dans la société postmoderne présente un débat récurrent sur une possible fossé générationnel entre personnes jeunes et âgés. Le postmodernisme, montée de l'individualisme et le relativisme, contraste aujourd'hui avec des besoins posés par la situation économique et la reformulation des priorités de valeur. Dans les circonstances actuelles, nous nous demandons si la crise mondiale n'aura pas homogénéise les valeurs des jeunes et vieux, puisque les deux ont vécu étapes de la socialisation dans un contexte de plus grande rareté et l'insécurité. Le but de cette étude est de comparer les similitudes et les différences dans l'appréciation des valeurs des deux cohortes, selon Rvs (Rokeach) échelle. Les résultats montrent des similitudes considérables dans les valeurs fondamentales qui privilégient les deux groupes. Il est également mis en évidence chez les jeunes un taux plus élevé du postmodernisme, car ils présentent une plus grande pluralité et diversification vers des valeurs individualistes et intrapersonnelles.

Mots clés: valeurs; postmodernisme; crise économique; étudiants; étudiants plus âgés. 
M. J. HERNÁNDEZ SERRANO, G. PARRA NIETO Y M. D. PÉREZ GRANDE

PRIORIZACIÓN DE VALORES EN ESTUDIANTES JÓVENES Y MAYORES EN EL CONTEXTO DE LA CRISIS ECONÓMICA

\section{INTRODUCCIÓN}

La recesión, la crisis financiera y las aceleradas transformaciones económicas y sociales de las últimas décadas plantean en las sociedades occidentales nuevos retos y demandas educativas a diferentes niveles (Damme y Karkkainen, 2011). Las repercusiones asociadas a la vivencia de una etapa de escasez e inseguridad están comportando, tanto para educandos como para educadores, cierta reformulación de prioridades, que probablemente esté trascendiendo también al plano de las prioridades axiológicas. Si los valores son preferencias colectivas e individuales que aparecen en un contexto social y cultural, también contribuyen a su regulación (Boudon y Bourricaud, 1990), y a que dicho contexto tenga un rol moderador decisivo (Bardi y Goodwin, 2011). La sociedad y la cultura asumen la función de proveer el contexto a partir del cual las personas eligen los valores, las formas deseables de conducta y las finalidades de la existencia (Reynolds, 1984), así se erigen las preferencias que se arraigan en nuestras creencias internas para regular el pensamiento y la acción (Rokeach, 1968, 1973). De esta interdependencia con el contexto deriva el carácter dinámico de los valores (Kemmis, 2000) y, con ello, la posibilidad de que los cambios macrosociales puedan provocar modificaciones en las preferencias, en las prioridades y en los valores, con consecuencias significativas para el desarrollo social y la educación.

Es preciso tener en cuenta que, en el análisis de esa nueva dirección epistemológica y axiológica (Edwards, 2006), no sólo hallamos los factores contextuales, globales o socioeconómicos que definen decisivamente las elecciones vitales, sino también las tensiones y controversias aún no resueltas del paso del modernismo al postmodernismo (Gur-Ze'ev, 2007; Wain, 2008; Todd, 2009; Brenneman y Margonis, 2012), que se traducen en un cambio de valores materialistas a postmaterialistas, y donde se insiste en que las prioridades de un individuo son el reflejo del contexto socioeconómico en el que ha vivido, especialmente durante las primeras etapas de su existencia, previas a la edad adulta (Inglehart, 1977, 1981, 1990, 1991, 1995, 1997a, 1997b, 2000; Inglehart y Abramson, 1994; Inglehart y Klingemann, 1996; Inglehart y Welzel, 2005). De tal forma que, siguiendo la hipótesis de escasez de Inglehart (Inglehart y Welzel, 2005), cuando se han vivido épocas de escasez, se priorizan los valores asociados a la seguridad económica y personal, ya que se otorga un mayor valor subjetivo a situaciones deseables pero escasas en el contexto social predominante, y según la hipótesis de socialización de este mismo autor, esta priorización se mantendría incluso aunque más tarde se vivieran épocas de bienestar, especialmente para los valores más fundamentales de un individuo, que dependerían de las condiciones que predominaban durante los años en que la persona se estaba socializando.

De acuerdo a estas premisas, y a algunas investigaciones recientes donde se empieza a concluir cómo los momentos y contextos de transición están influyendo en la priorización de valores de los jóvenes, que parecen estar regresando a valores relacionados con la seguridad y la estabilidad (Myyry, Juujarvi y Pesso, 2013; Bardi, Buchanan, Goodwin, Slabu y Robinson, 2014; Tulviste y Tamm, 2014), 
este estudio toma como punto de partida el análisis de la dirección que toma el cambio de valores en el panorama postmoderno contemporáneo, marcado por un contexto socioeconómico de inestabilidad. Más concretamente, nos preguntamos si dicho contexto económico está produciendo un cambio de prioridades y preferencias, en dirección a la prevalencia de valores de corte tradicional, propios de la modernidad, conduciendo a que los jóvenes - quienes se encuentran en periodo de socialización y viven una situación de escasez- vuelvan hacia valores materialistas, de supervivencia económica y seguridad personal, que se habían difuminado en las épocas de gran bonanza económica. Nos preguntamos también si, en caso de que esto suceda, sus valores podrían estar acercándose a los que vivieron sus mayores, dos generaciones atrás, también socializados en épocas de crisis y escasez económica.

En el presente artículo comenzamos por revisar las valoraciones teóricas en torno al cambio de valores modernistas a postmodernistas, para reflexionar sobre el posible efecto de la crisis económica y su posible impacto sobre el repunte del materialismo. De manera complementaria, en la parte empírica del artículo, analizamos la priorización de valores que realizan estudiantes universitarios jóvenes y mayores para contrastar la existencia o no de diferencias intergeneracionales en la priorización de valores modernistas y posmodernistas.

\section{Planteamiento Del estudio}

\subsection{Diferencias en la valoración de los cambios sociales}

Las teorías acerca de la transformación de la sociedad postmoderna son ampliamente aceptadas desde los diferentes ámbitos de las ciencias sociales. Hay un claro consenso acerca de los factores históricos determinantes que han transformado radicalmente la sociedad; se ha pasado de una sociedad basada en la producción materialista a otra basada en el conocimiento y la información, lo que necesariamente lleva a cambios significativos respecto a la vida social y la educación (Martínez, Esteban y Buxarrais, 2011; Rumbo, 2013; Kemmis, 2000) y a un notable cambio de valores que se define como una transición del mundo moderno al postmodernismo (Beck, 1998; Lipovetski y Charles, 2006; Tranter y Western, 2010; Bauman, 2001; Alonso, Jiménez-López, García-Vargas y Gil-RoalesNieto, 2013). Existe, sin embargo, un menor acuerdo y una cierta polémica sobre su valoración.

Si las ideas modernas estaban basadas en la búsqueda de "la verdad" y la fe en la ciencia para conseguir el progreso, las ideas postmodernas defienden «diferentes verdades", es decir, la ausencia de verdades absolutas, el relativismo, la importancia del momento presente y la fragmentación del conocimiento. Desde una visión positiva, las personas se han liberado de estar pendientes de la mera supervivencia y de las dependencias materiales, lo que conduce a incrementar valores de autoexpresión y emancipación, libertad de elección, autonomía e igualdad, que 
favorecen una cultura humanista creativa y centrada en las personas (Inglehart y Wetzel, 2006; Fernández, 2010). Desde una visión más crítica y pesimista, otros autores sostienen que el postmodernismo ha traído consigo valores superficiales e inconsistentes (Colom y Melich, 1993; Fullat, 2003; Barrio, 2008), que el excesivo relativismo que promueven resulta deseducativo para los jóvenes y que los valores de la educación postmoderna carecen de relieve; son planos, fugaces, provocando así dudas y perplejidades en las personas jóvenes (Fullat, 2003). Además, la pluralidad y saturación de información en la sociedad postmoderna, unidas al síndrome de la impaciencia que padece nuestra sociedad actual, no dejarían espacio ni tiempo para la reflexión personal, para pasar de la información al conocimiento (Perrenoud, 2001; Santos, 2010), lo que impediría un adecuado posicionamiento cuando se trata de elegir los propios valores (Bauman, 2007).

En este sentido, la educación postmoderna ha sido definida como aquella que no admite la universalidad de verdades, valores o prácticas y que promueve valores y vínculos sociales cada vez más "líquidos» y menos comprometidos (Bauman, 2001; Gervilla, 2002, 2011; Laudo y Prats, 2013). Se critica que esta educación pretende eliminar cualquier práctica directiva, y se encuentra cerca de las ideologías del "dejar hacer» y la desescolarización, provocando así una superficialidad desencantada y un relativismo ante tantas opciones y caminos vitales, que originan desorientación, perplejidad, desmotivación y falta de compromiso (Barrio, 2008; Fullat, 2003). Bauman (2005, 2007) y Sennett (2000) argumentan, además, que el proyecto de vida postmodernista tiene para los educandos una menor coherencia interna, no dirigiéndoles hacia finalidades específicas, sino a metas fragmentadas, dependientes de voluntades individuales, lo que al mismo tiempo fomenta un individualismo preocupante (Lipovetsky, 1986; Elzo y Castiñeira, 2011). Otros autores destacan sobre todo la dificultad de educar en valores en un mundo tan abierto y plural, considerando que resultan imprescindibles el diálogo, el consenso y la colaboración de todos los agentes socializadores (García del Dujo y Mínguez, 2011; Martínez, Esteban y Buxarrais, 2011).

El correlato de argumentos, en uno y otro sentido, no hace más que evidenciar lo que se ha denominado la doble cara de la postmodernidad. Por una parte, la pluralidad, la diversidad y la libertad conducen a plantear valores y discursos sumamente sugerentes y atractivos en ámbitos culturales y educativos; por ejemplo, la superación del etnocentrismo, los movimientos a favor de la paz, el respeto al medio ambiente, la igualdad de género, argumentos con planteamientos pedagógicamente enriquecedores. Pero, por otra, se señalan también las degradaciones culturales y cívicas que ha traído consigo la postmodernidad; por ejemplo, el aumento de las desigualdades, el exagerado individualismo y relativismo o el excesivo enriquecimiento de algunos estratos sociales a costa de otros (Mayos, 2011; Rumbo, 2013).

De hecho, una de las polémicas en torno a la postmodernidad es la que se origina al plantear si el cambio de valores se produce como consecuencia del fracaso del modernismo, o si, por el contrario, supone una continuación del mismo, 
es decir, se limita a una expansión de los valores básicos que ya se encontraban en el materialismo. No hay acuerdo en este aspecto. Algunos autores argumentan que no se trata de una sustitución, sino de una coexistencia de valores (Gil RoalesNieto y Segura, 2010; Klein y Pötschke, 2000). Inglehart y Welzel (2006) ya habían afirmado que, por una parte, la industrialización promueve la decadencia de los valores tradicionales, pero, por otra, de forma simultánea, hay una permanencia de las tradiciones en la vida de las personas. Del mismo modo, Bericat (2003) sostiene que en la postmodernidad avanzamos hacia una "hipermodernización" al mismo tiempo que siguen presentes procesos de "tradicionalización». Aunque ambos procesos pueden parecer contradictorios, algunos autores señalan que, en realidad, son complementarios (Díez Nicolás, 2011; Fernández, 2010; Rumbo, 2013), es decir, que los principales valores continúan en las preferencias de los sujetos, aunque sea en otro orden de importancia o utilidad.

La diversidad de planteamientos, contradicciones y polémicas que se mantienen en la literatura científica acerca del cambio de valores en nuestras sociedades dan cuenta de su complejidad, no existiendo respuestas sencillas. Además, como afirma Reimer (1988), la distinción entre materialismo y postmaterialismo puede resultar demasiado rígida para captar las complejidades de los valores de las personas. Sí entendemos, al menos, que se debe hacer una lectura distinta del postmodernismo: una lectura no lineal, que no identifique únicamente la postmodernidad con el individualismo o el relativismo, pues estos valores coexisten con la preocupación por el grupo, el respeto a las diferencias y los límites de los derechos humanos. Desde una lectura que privilegie la continuidad conviene situar el materialismo y el postmaterialismo como etapas donde concurren, de acuerdo a la teoría de Rokeach (1973), tanto valores individualistas como prosociales. Este autor ha diferenciado dos grupos de valores, los instrumentales, relativos a modos de conducta necesarios para convivir, y los finalistas, referidos a estados deseables. En ambos grupos se pueden distinguir valores que responden tanto a metas intrapersonales -individuales o deseables para la realización personal- como interpersonales -para el ámbito relacional y social del individuo-.

Esta distinción puede aportar interesantes comparaciones, como se pretende desde este estudio, a la hora analizar los cambios de valores y de preferencias de unas generaciones a otras, ya que las metas de bienestar o supervivencia, la priorización de libertades o de necesidades, de autorrealización o de seguridad adquieren otra interpretación cuando se estudian desde la relevancia para uno mismo y para la convivencia con los demás.

\subsection{Las diferencias intergeneracionales. El impacto de la crisis económica en el postmaterialismo}

Cuando se analizan los cambios sociales, de acuerdo a las hipótesis de Inglehart y otros autores (Inglehart, 1977, 1991; Inglehart y Welzel, 2005; Norris e Inglehart, 2011), se pueden establecer diferencias generacionales, entendiendo 
que las cohortes más jóvenes serían más postmaterialistas que las mayores, al no haber vivido épocas de escasez y haber experimentado un mayor desarrollo social y económico, y a la inversa. La estabilidad y la bonanza económica habrían sido interiorizadas especialmente por jóvenes que, además de haber vivenciado más seguridad que los mayores, no poseen -debido a su menor recorrido vital-visiones o hábitos demasiado arraigados, lo que les llevaría a defender valores de autoexpresión y liberación como la libertad, la igualdad de género o la participación (Fernández, 2010). Por otra parte, las generaciones más jóvenes han crecido bajo la influencia de las tecnologías, que implican posibilidades de acceso a gran cantidad de información de forma rápida y cambiante. Colom y Melich (1993) ya afirmaron que tal sobreabundancia de información sin un buen grado de reflexión podría llevar a los jóvenes a conclusiones y consistencias más transitorias e instantáneas. Por ello, en esta etapa vital resulta imprescindible el papel de la educación para la construcción de valores apropiados con el fin de hacer frente a los rápidos cambios sociales.

Los valores postmaterialistas experimentaron un aumento constante desde 1981 a 2005 en países emergentes y en países desarrollados (Díez Nicolás, 2011). A partir de esa fecha y debido al aumento de problemas relacionados con la inseguridad social y personal (el terrorismo, el narcotráfico, la delincuencia, las bandas criminales o el incremento de la inmigración), según este autor, la tendencia empezó a invertirse. Un factor clave añadido en esta transposición de valores es el fenómeno de la crisis económica internacional, que afecta profundamente a los distintos sectores sociales y generacionales. Por ejemplo, si comparamos los sondeos del cis de 2007 y 2014, antes y tras el impacto de la crisis, encontramos que el paro ha pasado de ser considerado como preocupación principal por el $35 \%$ de los encuestados ${ }^{1}$, a serlo para el $76,8 \%{ }^{2}$, habiendo aumentado también las personas preocupadas por la corrupción (del 0,7\% al 38,8\%), sin embargo, ha disminuido la preocupación por la inmigración (del 29,2\% al 3,1\%) y por el terrorismo (del 35,4\% al $0,5 \%$ ). Los datos reflejan un aumento del nivel de inestabilidad e inseguridad en el aspecto económico. ¿Este cambio en las preocupaciones de la ciudadanía marcaría un cambio de valores? ¿Nos retraería a valores de escasez y supervivencia, según las hipótesis de Inglehart? Especialmente los jóvenes, ¿están viviendo situaciones de escasez, con la misma intensidad que adultos de las generaciones anteriores? Si esto es así, ¿se estaría eliminando la brecha intergeneracional, al mostrar los jóvenes una mayor preferencia por los valores materialistas?

Algunas investigaciones recientes empezarían a confirmar cómo estos cambios profundos estarían llevando a una disminución de lo que podríamos denominar índice de postmaterialismo, respecto a la menor priorización de valores postmaterialistas o propios del postmodernismo, tanto por parte de jóvenes, como de mayores. La investigación de Fernández (2010), donde se comparan varias comunidades

1. Barómetro español del Centro de Investigaciones Sociológicas (CIS), septiembre de 2007.

2. Barómetro del CIs, junio de 2014 . 
autónomas, confirma un claro repunte de valores materialistas en la población de Andalucía, más llamativo porque se produce en personas con un alto nivel de formación. Este viraje se explicaría por el aumento del desempleo del personal cualificado en la comunidad andaluza, con la tasa de paro más alta de España. Al comparar con la población catalana, encuentra que en esta última, en cambio, los valores materialistas han disminuido, atribuyendo este dato a un mayor desarrollo socioeconómico. Por otro lado, en el estudio intergeneracional llevado a cabo por Alonso et al. (2013) se encuentra que la diferencia más significativa entre mayores y jóvenes se produce en los valores relacionados con la salud y el afecto, concluyendo que pueden ser más importantes las condiciones personales y necesidades vitales que los cambios sociales. Ciertamente, otras investigaciones confirman el peso de variables como el lugar de origen, la cultura local, el nivel de estudios, las circunstancias vitales y el género (Lila, Musito y Buelga, 2000; Fernández, 2010; Yagüe et al., 2013), que modularían las preferencias de valores y los modelos deseables de conducta.

Por tanto, es importante tener en cuenta que los sistemas de valores, en tanto que tendencias individuales o sociales, no sólo dependen de los cambios macrosociales, sino también de los cambios vitales. La conformación y priorización de valores actúa de manera dinámica y puede cambiar con el tiempo y según las necesidades que se imponen en cada etapa vital. El estudio del desarrollo moral (Piaget, 1932; Kohlberg, 1976; Rest, Narvaez, Thomas y Bebeau, 2000; García y Pérez, 2005) y la psicología evolucionista (Buss y Hawley, 2011 y Yagüe, Sánchez, De Miguel y Gómez, 2013) confirmarían dichos cambios evolutivos en las prioridades de los valores, en lo que refiere al plano intergeneracional, e intersexos.

Todas estas consideraciones, así como los últimos resultados de las investigaciones que muestran el aumento de los valores materialistas, y menores diferencias entre jóvenes y mayores, nos ha llevado a plantearnos las siguientes preguntas de investigación:

- ¿Siguen los jóvenes priorizando en mayor medida que los mayores, valores postmaterialistas a pesar de la crisis económica y el descenso del bienestar social?

- ¿Qué tipo de valores presentan más diferencias o coincidencias entre jóvenes y mayores?

- ¿Qué diferencias aportan las variables sexo o nivel de estudios en la priorización de valores?

Tratando de responder a estas cuestiones se ha diseñado un estudio para analizar las preferencias axiológicas de un grupo de personas jóvenes y otro de mayores, en un entorno universitario, a través de la Escala de Valores de Rokeach que detallamos a continuación. Las diferencias se han analizado en función de la edad, el sexo y el nivel de estudios. 
M. J. HERNÁNDEZ SERRANO, G. PARRA NIETO Y M. D. PÉREZ GRANDE

PRIORIZACIÓN DE VALORES EN ESTUDIANTES JÓVENES Y MAYORES EN EL CONTEXTO DE LA CRISIS ECONÓMICA

\section{MÉTODO}

\subsection{La Escala de Valores de Rokeach}

Existe una gran variedad de instrumentos para el estudio de los valores individuales. Los procedimientos más habituales son tres: plantear al sujeto preguntas abiertas ante determinadas situaciones (Gil Roales-Nieto, 2009; Jiménez-López, 2011), pedirle que ordene una lista de valores según la importancia atribuida (Rokeach, 1973) o solicitarle que evalúe los diferentes valores de una lista (Inglehart y Abramson, 1994; Inglehart y Klingemann, 1996; Klein, Dülmer, Ohr, Quandt y Rosar, 2004). Cheng y Fleischmann (2010) revisaron diversas escalas de análisis y evaluación de valores llegando a la conclusión de que las más completas y adecuadas eran la Rokeach Value Survey (RvS) y la Schwart Value Survey (Svs). Si bien hay diferentes partidarios y detractores de cada procedimiento, podemos afirmar que cada método de evaluación ofrece potencialidades, además de que unos no excluyen a los otros sino que pueden complementarse (Ovadia, 2004; Beltrán, Torres, Beltrán y García, 2005; Daset, López y Suero, 2007; Sandoval, 2007; Penas, 2008).

Para nuestro estudio hemos optado por la Rvs de Rokeach (1973), instrumento que ha probado su eficacia psicométrica, siendo ampliamente aceptado por la comunidad científica (Lila, Musitu y Buelga, 2000; Schwartz y Sagie, 2000; Liubiniene, 2003; García y Pérez, 2005; Yagüe et al., 2013); aunque también haya recibido críticas metodológicas, especialmente por su procedimiento de clasificación ordinal (Tranter y Wesern, 2004, 2010).

La RVs presenta dos listas; la primera con 18 valores instrumentales (referentes a conductas reales o ideales) y la segunda con valores 18 finalistas (referentes a estados finales de existencia, que pueden ser de tipo intrapersonal o interpersonal) que el sujeto tiene que ordenar según la importancia que atribuye a cada valor (desde 1, valor más importante, a 18, valor menos importante) de manera anónima.

De acuerdo a nuestras preguntas de investigación y teniendo en cuenta que algunos valores no son fáciles de clasificar como modernistas o posmodernistas (Torcal, 1989), hemos situado en la Figura 1 los valores propuestos por Rokeach (1973) en la RSV a lo largo de un continuo. Se han separado los valores finalistas (abajo) de los instrumentales (arriba) y se han señalado en negrita los valores de dominio interpersonal, siendo el resto de carácter intrapersonal. Como punto de partida, observamos una mayor coincidencia de valores postmaterialistas - parte derecha de la figura- con intrapersonales, y de valores materialistas con interpersonales; otros valores se encuentran en el medio del continuo modernismo-postmodernismo, sin una clara orientación hacia uno u otro polo (por ejemplo amor, amistad, valentía). Como puede observarse, los valores finalistas resultan más fácilmente clasificables en modernistas o postmodernistas, mientras que muchos de los instrumentales quedan en medio del continuo. 
Figura 1. CONTINUO MATERIALISMO-POSTMATERIALISMO

EN LOS VALORES DE LA ESCALA RSV DE ROKEACH (1973)

\begin{tabular}{|c|}
\hline $\begin{array}{l}\text { Servicial } \\
\text { Obediencia } \\
\text { Perdón } \\
\text { Cortesía } \\
\text { Lógica } \\
\text { Limpieza }\end{array}$ \\
\hline
\end{tabular}

Honestidad

Amor Alegría
Responsabilidad
Valentía

Tolerancia

Ambición Independencia

MATERIALISMO

Salvación

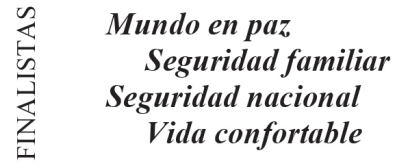

Capacidad Inteligencia

Imaginación
POSTMATERIALISMO

Libertad Placer

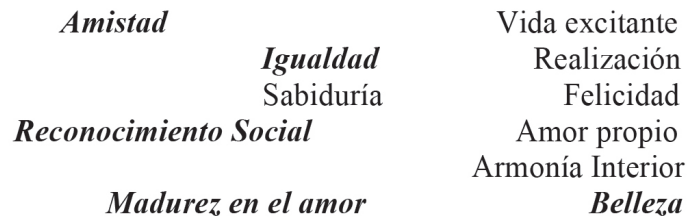

\subsection{Participantes}

El presente estudio forma parte de una investigación más amplia, transversal, que comenzó en 2011 y continúa en la actualidad. Para este trabajo se seleccionó una muestra (ver Tabla 1) de estudiantes universitarios jóvenes de las titulaciones en Educación (Pedagogía, Psicopedagogía, Educación Social y Magisterio), sujetos que por su edad hubieran vivido su juventud en el momento inicial de la crisis económica, en consonancia con el objetivo del estudio y las hipótesis de Inglehart. Y otra muestra de estudiantes mayores matriculados en el Programa Interuniversitario de la Experiencia de la misma Universidad, que hubieran vivido su juventud en un contexto de escasez e inestabilidad. En este último grupo se tuvo en cuenta, además, la variable nivel de estudios, considerando "con estudios» a las personas que tenían finalizados, como mínimo, estudios secundarios y "sin estudios» a aquellos por debajo de este nivel, con objeto de que dicha variable pudiera aportar diferencias relevantes en el análisis. 
M. J. HERNÁNDEZ SERRANO, G. PARRA NIETO Y M. D. PÉREZ GRANDE

PRIORIZACIÓN DE VALORES EN ESTUDIANTES JÓVENES Y MAYORES EN EL CONTEXTO DE LA CRISIS ECONÓMICA

TABla 1. Categorización de la muestra Del ESTUdio

\begin{tabular}{|c|c|c|c|c|c|c|}
\hline \multirow{2}{*}{\multicolumn{2}{|c|}{ GRUPO }} & \multirow{2}{*}{$n$} & \multicolumn{2}{|c|}{ EDAD } & \multicolumn{2}{|c|}{ SEXO } \\
\hline & & & RANGO & MEDIA & VARONES & MUJERES \\
\hline \multicolumn{2}{|c|}{ Jóvenes } & 166 & $\begin{array}{l}18-21 \\
\text { años }\end{array}$ & 21,5 & 82 & 84 \\
\hline \multirow{2}{*}{ Mayores } & $\begin{array}{c}\text { Con } \\
\text { estudios }\end{array}$ & 81 & $\begin{array}{l}55-72 \\
\text { años }\end{array}$ & 63,5 & 45 & 36 \\
\hline & $\begin{array}{c}\text { Sin } \\
\text { estudios }\end{array}$ & 67 & $\begin{array}{l}55-81 \\
\text { años }\end{array}$ & 66 & 26 & 41 \\
\hline \multicolumn{2}{|c|}{ TOTAL } & 314 & & & 153 & 161 \\
\hline
\end{tabular}

\subsection{Análisis de datos}

Para analizar los resultados -y las diferencias existentes entre ambos grupos, jóvenes y mayores, con estudios y sin estudios, hombres y mujeres-, se calculó la moda de cada uno de los valores propuestos, por tratarse de variables ordinales, de acuerdo a otros estudios (Yagüe et al., 2013), estableciendo así el orden de rango de los 36 valores analizados. Entre aquellos valores que obtuvieron la misma moda, se usó como criterio la puntuación de la mediana y la desviación del primer y tercer cuartil. El procesamiento estadístico de los datos para el análisis de las frecuencias y la comparación cuantitativa inter- e intragrupal (test Kruskal Wallis) se realizó con el paquete estadístico SPSS v.18.

\section{Resultados Y Discusión}

Como se aprecia en la Tabla 2, existen muchas coincidencias en los valores instrumentales más estimados (en primer, segundo y tercer lugar) por el grupo de estudiantes jóvenes y por el de los mayores, lo que concuerda con los datos de otros estudios (Gil Roales-Nieto y Segura, 2010; Alonso et al., 2013).

Los valores instrumentales más priorizados por todos los grupos son: honestidad, responsabilidad, alegría y tolerancia. Los dos primeros (honestidad y responsabilidad) coinciden como prioritarios en las investigaciones de Reynolds (1984), de García y Pérez (2005), en la revisión de Martí y Palma (2010), en la de Yagüe et al. (2013) y en la de Ryan, Schruder y Robinson (2013). Este último estudio añade amor y lealtad a las prioridades.

Según nuestros datos, los jóvenes muestran un índice más elevado de postmodernismo. Por una parte, presentan una mayor dispersión, diversidad y pluralidad de prioridades; mientras que una gran parte del grupo de mayores se concentra en una cantidad limitada de valores prioritarios, el grupo de jóvenes eligen un mayor 
número de ellos (por ejemplo, son priorizados también, amor, capacidad e independencia). Por otra, los valores elegidos por los jóvenes tienen una connotación más postmaterialista. Estos datos responderían a una de las preguntas que nos planteábamos en torno a la existencia de un mayor nivel de postmodernismo en los jóvenes, entendido sobre todo como una mayor diversidad y relativismo en sus valores prioritarios, pluralidad que también se refleja en los análisis aportados por otros estudios (Yagüe et al., 2013).

En relación a la variable nivel de estudios encontramos que el grupo de mayores con estudios considera como valor prioritario la inteligencia (en el apartado de valores finalistas veremos que también consideran prioritario el valor sabiduría). Posiblemente, quienes priorizan estos valores se encuentran más inclinados a realizar el esfuerzo de estudiar.

TABLA 2. COMPARATIVA DE VALORES INSTRUMENTALES PRIORIZADOS EN LOS GRUPOS ${ }^{3}$

\begin{tabular}{|c|c|c|c|}
\hline & 1. ESTUdiantes JÓVENES & 2. ESTUDIANTES MAYORES & 3. MAYORES SIN ESTUDIOS \\
\hline $1 .^{\circ}$ & $\begin{array}{c}\text { Tolerancia, Alegría, } \\
\text { Amor }\end{array}$ & $\begin{array}{l}\text { Honestidad, } \\
\text { Inteligencia }\end{array}$ & Honestidad \\
\hline $2 .^{\circ}$ & $\begin{array}{l}\text { Honestidad, } \\
\text { Independencia }\end{array}$ & Tolerancia, Alegría & Responsabilidad, Alegría \\
\hline $3 .^{\circ}$ & $\begin{array}{l}\text { Capacidad, } \\
\text { Responsabilidad }\end{array}$ & Responsabilidad & Tolerancia \\
\hline \multicolumn{4}{|l|}{$\cdots$} \\
\hline $16 .^{\circ}$ & Limpieza & Limpieza & $\begin{array}{c}\text { Lógica, } \\
\text { Autocontrol }\end{array}$ \\
\hline $17 .^{\circ}$ & Lógica & Lógica & $\begin{array}{l}\text { Obediencia, } \\
\text { Competencia }\end{array}$ \\
\hline $18 .^{\circ}$ & $\begin{array}{c}\text { Obediencia, Ambición, } \\
\text { Imaginación }\end{array}$ & $\begin{array}{c}\text { Obediencia, Ambición, } \\
\text { Imaginación }\end{array}$ & Ambición, Imaginación \\
\hline
\end{tabular}

En cuanto a los valores instrumentales menos relevantes (estimados en los últimos lugares: $18 .^{\circ}, 17 .^{\circ}$ o $16 .^{\circ}$ ) hay un elevado grado de acuerdo entre todos los grupos: ambición, imaginación, lógica y obediencia. Estos datos se contraponen al estudio de Yagüe et al. (2013), que encuentran entre los jóvenes la ambición y la imaginación como valores altamente estimados. Asimismo,

3. Los valores en negrita son aquellos en los que coinciden las prioridades de jóvenes y mayores. 
se constata en los sujetos con mayor nivel de estudios que el valor limpieza queda en las últimas posiciones. Es posible que a partir de determinado nivel social y de estudios la limpieza se dé por sentada y no constituya un valor anhelado. Los mayores sin estudios eligen en cambio el autocontrol y la competencia como valores menos apreciados.

Es preciso subrayar que, cuando hablamos de los últimos lugares en la escala de estimación, no podemos entender que las personas de la muestra no atribuyan ninguna importancia a dichos valores, sino que, dentro de los valores presentados, estos son los que para ellas tienen una prioridad menor. También hay que señalar que algunos términos pueden tener connotaciones y significados diferentes en distintas culturas. Por ejemplo, es posible que el valor ambición se encuentre entre los menos valorados debido a que en nuestro contexto cultural los términos ambición y ambicioso mantienen una connotación negativa o peyorativa, de forma que incluso podría llegar a considerarse un contravalor. Por el contrario, en otros contextos, como el anglosajón y el americano, la ambición es entendida como un término positivo, relacionado con la capacidad de superación y el alcance de metas propias (Rokeach, 1979); por ello, en las investigaciones anglosajonas suele ser un valor mucho más estimado (véase, por ejemplo, Reynolds, 1984).

Es en los valores finalistas donde encontramos mayores diferencias en las escalas de valores de los grupos (ver Tabla 3). En el análisis intercohorte de algunos valores, hallamos algunas preferencias opuestas. Ejemplo de ello es la alta priorización del valor vida excitante (un valor postmodernista) entre los jóvenes, escasamente valorado entre los mayores, o la valoración de un mundo en paz y seguridad familiar (valores modernistas), en los que sucede justamente lo contrario, tal y como encontraron también en su trabajo Yagüe et al. (2013). Estas discrepancias confirmarían también un mayor índice de postmaterialismo en los jóvenes, posiblemente, siguiendo las hipótesis de Inglehart, como consecuencia del contexto marcado por la etapa histórica que les ha tocado vivir (condiciones socioeconómicas menos duras y ausencia de la experiencia de vivir una guerra/postguerra). Probablemente las diferencias intercohorte podrían ser también explicadas por la discrepancia de necesidades en cada una de las etapas vitales. Como ha afirmado Torcal (1989), muchas de las diferencias entre jóvenes y mayores se explican más por el ciclo vital que por el cambio sociocultural; mientras en la juventud se tiende a buscar experiencias nuevas y motivantes como parte del proceso de desarrollo y crecimiento, en la madurez tiende a valorarse una mayor estabilidad y tranquilidad.

Los resultados son similares a los que encuentra Hofmann (2009) en su trabajo con una muestra de jóvenes universitarios, en los que se destacan como prioritarios la libertad y el respeto por sí mismo (valores postmodernistas). Por otra parte, Ryan, Schruder y Robinson (2013) señalan también como valores prioritarios en jóvenes universitarios la amistad y la libertad, añadiendo la seguridad familiar que, sin embargo, en nuestro estudio es más valorada por personas mayores. Este mayor índice de postmodernismo entre los jóvenes no quiere decir que entre las personas mayores no se prioricen también valores postmaterialistas; de hecho, entre 
las coincidencias intergeneracionales aparecen como más estimados los valores finalistas postmaterialistas libertad y felicidad.

Entre los valores menos priorizados, hay de nuevo claras coincidencias entre jóvenes y mayores respecto a valores tradicionales, tanto materialistas (salvación), como postmaterialistas hedonistas (mundo de belleza). Por otra parte, destaca la baja estimación del placer entre los mayores, coincidiendo asimismo con la investigación de Hofmann (2009), en la que aparecen como menos estimados entre los jóvenes la salvación y el mundo de belleza, así como la seguridad nacional y mundo en paz.

TABLA 3. COMPARATIVA DE VALORES FINALISTAS PRIORIZADOS EN LOS GRUPOS ${ }^{4}$

\begin{tabular}{|c|c|c|c|}
\hline & 1. ESTUDIANTES JÓVENES & 2. ESTUDIANTES MAYORES & 3. MAYORES SIN ESTUDIOS \\
\hline $1 .^{\circ}$ & Felicidad & $\begin{array}{l}\text { Felicidad, } \\
\quad P a z\end{array}$ & $\begin{array}{l}\text { Felicidad, } \\
\qquad P a z\end{array}$ \\
\hline $2 .^{\circ}$ & Libertad & $\begin{array}{c}\text { Libertad, } \\
\text { Armonía interior }\end{array}$ & $\begin{array}{c}\text { Libertad, } \\
\text { Seguridad Familiar }\end{array}$ \\
\hline $3 .^{\circ}$ & $\begin{array}{l}\text { Vida excitante, } \\
\text { Amistad }\end{array}$ & $\begin{array}{c}\text { Seguridad Familiar } \\
\text { Sabiduría }\end{array}$ & Vida confortable \\
\hline \multicolumn{4}{|l|}{$\ldots$} \\
\hline $16 .^{\circ}$ & $\begin{array}{l}\text { Seguridad nacional, } \\
\text { Reconocimiento social }\end{array}$ & Belleza & Amor maduro, Placer \\
\hline $17 .^{\circ}$ & Belleza & Placer & Vida excitante \\
\hline $18 .^{\circ}$ & Salvación & Salvación & Belleza, Salvación \\
\hline
\end{tabular}

Con el fin de encontrar diferencias estadísticamente significativas en las comparaciones intercohorte, intersexo y por nivel de estudios se realizó la prueba Kruskal Wallis Test (con los valores de $\chi^{2}$ ). En las siguientes tablas ( 4 y 5 ) presentamos los resultados señalando las diferencias que son estadísticamente significativas ${ }^{5}$.

Los porcentajes obtenidos indican que son menores las discrepancias intercohorte en lo que se refiere a valores instrumentales; de hecho solo hallamos diferencias significativas de estimación en el 38.88\% de los valores (ver Tabla 4). En cambio, en valores finalistas, este porcentaje aumenta hasta el 66.6\% (ver Tabla 5). Esta tendencia se repite en las diferencias por nivel de estudios: $38.88 \%$ en

4. Los valores en negrita son aquellos en los que coinciden las prioridades de jóvenes y mayores.

5. Valor de F: ${ }^{*} \mathrm{p} \leq .05 ; * * \mathrm{p} \leq .01 ;{ }^{* * *} \mathrm{p} \leq .001$. 
M. J. HERNÁNDEZ SERRANO, G. PARRA NIETO Y M. D. PÉREZ GRANDE

PRIORIZACIÓN DE VALORES EN ESTUDIANTES JÓVENES Y MAYORES EN EL CONTEXTO DE LA CRISIS ECONÓMICA

instrumentales y $44.44 \%$ en finalistas; y en las discrepancias intersexo: $16.66 \%$ y $33.33 \%$ respectivamente.

TABla 4. COMPARATIVA DE DifERENCIAS POR NIVEL DE ESTUDiOS, GÉNERO Y EDAD EN VALORES INSTRUMENTALES

\begin{tabular}{|c|c|c|c|c|c|}
\hline & & $\begin{array}{l}=314 . \text { Con estudios superiores } N=240 ; \\
\text { Sin estudios } N=74 \\
\text { Hombres } N=153 ; \text { Mujeres } N=161 \\
\text { Jóvenes } N=118 ; \text { Mayores } N=196\end{array}$ & $\begin{array}{l}\text { Nivel de } \\
\text { Estudios }\end{array}$ & Intersexo & Intercohorte \\
\hline & & TOLERANCIA (apertura mental) & 0.05 & $5.73^{* * *}$ & 2.15 \\
\hline & & HONESTIDAD (sinceridad, veracidad) & 0.09 & 1.02 & 0.48 \\
\hline & & AMOR (ser afectuoso, tierno) & $4.18^{*}$ & 0.32 & $22.87^{* * * * * *}$ \\
\hline & z & ALEGRÍA (jovialidad, buen humor) & 0.15 & 0.42 & $11.74^{* * * *}$ \\
\hline & 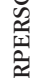 & $\begin{array}{l}\text { SERVICIO (preocuparse del bienestar } \\
\text { de los otros) }\end{array}$ & 0.16 & 1.23 & $7.85^{*}$ \\
\hline & $\underline{E}$ & OBEDIENCIA (sumisión, respeto) & $13.16^{* * * * * *}$ & 0.75 & $6.87 * *$ \\
\hline & & PERDÓN (estar dispuesto a perdonar) & 3.68 & 2.60 & 1.43 \\
\hline 起 & & CORTESÍA (ser atento, educado) & 3.22 & 0.09 & 3.50 \\
\hline 还 & & RESPONSABILIDAD (ser serio, fidedigno) & 0.00 & 2.25 & $5.09^{*}$ \\
\hline 聯 & & CAPACIDAD (competencia, efectividad) & $8.16^{* * *}$ & 1.33 & 0.01 \\
\hline 踢 & & IMAGINACIÓN (atrevimiento, creatividad) & $10.90^{* * * * *}$ & 0.02 & $8.51^{* * *}$ \\
\hline 点 & & $\begin{array}{l}\text { INDEPENDENCIA (seguridad, } \\
\text { autosuficiencia) }\end{array}$ & 0.84 & 3.19 & \\
\hline & 充 & INTELIGENCIA (capacidad intelectual) & $5.98^{*}$ & 2.73 & 0.61 \\
\hline & $\underset{\infty}{\infty}$ & LÓGICA (consistencia, racionalidad) & $8.58^{* * *}$ & 3.38 & $4.16^{*}$ \\
\hline & 定 & VALENTÍA (defender las ideas) & 0.02 & 0.02 & 0.14 \\
\hline & 艺 & LIMPIEZA (ser cuidadoso, ordenado) & $20.59^{* * * * *}$ & $5.13^{* * *}$ & $16.97^{* * * * *}$ \\
\hline & & $\begin{array}{l}\text { AMBICIÓN (trabajar duramente, tener } \\
\text { aspiraciones) }\end{array}$ & 0.26 & $18.67^{* * * *}$ & 3.52 \\
\hline & & $\begin{array}{l}\text { AUTOCONTROL (ser autodisciplinado, } \\
\text { moderado) }\end{array}$ & 0.96 & 0.24 & 2.81 \\
\hline
\end{tabular}

Respecto a los valores instrumentales (ver Tabla 4), observamos que solamente el valor limpieza -uno de los menos priorizados globalmente- ofrece diferencias significativas intercohorte, intersexo y nivel de estudios. Resulta menos priorizado por los jóvenes (mediana, Me $=15$ ) que por los mayores, especialmente por los que no tienen estudios $(\mathrm{Me}=10)$. En cuanto a las diferencias intersexo, observamos una 
mayor estimación por parte de las mujeres frente a los hombres, lo cual coincide con el estereotipo femenino de mayor preocupación por la limpieza.

Atendiendo a las diferencias intercohorte, apreciamos un $55.55 \%$ de discrepancias en los valores instrumentales de orientación interpersonal (ver Tabla 3). Las más significativas se producen en los valores de amor y alegría, más estimados por la cohorte de jóvenes con medianas de 5 y 4 , frente a la cohorte de adultos mayores con una posición en sus medianas de 8 y 6 respectivamente, lo que confirma los resultados encontrados por Yagüe et al. (2013). Con el valor obediencia sucede lo contrario, resultando más priorizado por el grupo de mayores.

En cuanto a los valores instrumentales intrapersonales, el porcentaje de discrepancia es menor y se sitúa en el 33.33\%. La imaginación es el valor donde se produce una diferencia más significativa ya que es estimado en los últimos lugares por los mayores $(\mathrm{Me}=16)$ frente al lugar intermedio en que lo sitúan los jóvenes $(\mathrm{Me}=10)$.

Por nivel de estudios, los porcentajes varían entre un 22.22\% (valores instrumentales de orientación interpersonal, denominados por Rokeach morales) y un $55.55 \%$ (valores instrumentales intrapersonales, o denominados de competencia). El valor obediencia es el que presenta mayores diferencias, siendo escasamente estimado por las personas con estudios $(\mathrm{Me}=15)$, y situado en una posición intermedia por el grupo de personas sin estudios $(\mathrm{Me}=11.5)$.

En cuanto a los valores considerados intrapersonales, se observan diferencias significativas en imaginación, capacidad y lógica. La imaginación es escasamente priorizada por las personas con estudios $(\mathrm{Me}=15)$, mientras que las personas sin estudios la sitúan en lugares medios $(\mathrm{Me}=11.5)$. En cambio, los valores de lógica y capacidad son más valorados por el grupo de personas con estudios.

Centrándonos en la variable sexo, observamos que se presentan escasas diferencias significativas entre hombres y mujeres; un $1.11 \%$ en valores instrumentales interpersonales y un $22.22 \%$ en intrapersonales (Tabla 3 ). Se destaca el valor ambición, que resulta curiosamente más considerado por el sexo femenino en nuestra muestra $(\mathrm{Me}=10)$, frente al masculino $(\mathrm{Me}=14)$. Este dato no coincide con los estudios de Martí y Palma (2010) y de Yagüe et al. (2013), en los que se encontró este valor más priorizado por los varones.

En cuanto a los valores finalistas, se obtuvieron diferencias significativas entre los tres grupos analizados en los valores vida excitante, madurez en el amor y amistad verdadera (ver Tabla 5). 
M. J. HERNÁNDEZ SERRANO, G. PARRA NIETO Y M. D. PÉREZ GRANDE

PRIORIZACIÓN DE VALORES EN ESTUDIANTES JÓVENES Y MAYORES EN EL CONTEXTO DE LA CRISIS ECONÓMICA

TABla 5. COMPARATIVA DE DifERENCIAS POR NIVEL DE ESTUdios, GÉNERO Y EDAD EN VALORES FINALISTAS

\begin{tabular}{|c|c|c|c|c|c|}
\hline \multicolumn{3}{|c|}{$\begin{array}{c}N=314 . \text { Con estudios superiores } N=240 \\
\text { Sin estudios } N=74 \\
\text { Hombres } N=153 ; \text { Mujeres } N=161 \\
\text { Jóvenes } N=118 ; \text { Mayores } N=196\end{array}$} & \multirow{2}{*}{$\begin{array}{l}\text { Nivel de } \\
\text { Estudios } \\
14.65^{\text {***** }}\end{array}$} & \multirow{2}{*}{$\begin{array}{c}\text { Intersexo } \\
0.53\end{array}$} & \multirow{2}{*}{$\begin{array}{c}\text { Intercohorte } \\
0.01\end{array}$} \\
\hline \multirow{18}{*}{ 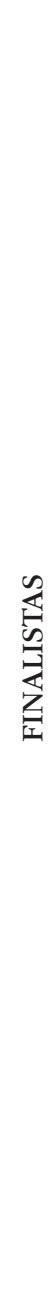 } & & UNA VIDA CONFORTABLE (próspera) & & & \\
\hline & & $\begin{array}{l}\text { UN MUNDO EN PAZ (sin guerras, } \\
\text { ni conflictos) }\end{array}$ & $6.89 * *$ & 0.05 & $40.69 * * *$ \\
\hline & & $\begin{array}{l}\text { UN MUNDO DE BELLEZA (natural y } \\
\text { artística) }\end{array}$ & 3.52 & 0.84 & 0.42 \\
\hline & 安 & $\begin{array}{l}\text { IGUALDAD (hermandad, iguales } \\
\text { oportunidades) }\end{array}$ & 0.12 & 1.11 & 0.77 \\
\hline & 赵 & $\begin{array}{l}\text { SEGURIDAD FAMILIAR (asegurarse el ser } \\
\text { amado) }\end{array}$ & 2.16 & 0.73 & $4.45^{* *}$ \\
\hline & $\underline{\underline{Z}}$ & $\begin{array}{l}\text { MADUREZ EN EL AMOR (sexual } \\
\text { y espiritualmente) }\end{array}$ & $11.00 * * * *$ & $4.01 *$ & $40.66^{* * * *}$ \\
\hline & & $\begin{array}{l}\text { SEGURIDAD NACIONAL (protección frente } \\
\text { a ataques) }\end{array}$ & $31.24^{* * * * *}$ & 0.29 & $42.55 * * *$ \\
\hline & & $\begin{array}{l}\text { RECONOCIMIENTO SOCIAL (respeto } \\
\text { y admiración) }\end{array}$ & 0.42 & $5.26^{* * *}$ & 3.10 \\
\hline & & AMISTAD VERDADERA (compañerismo) & $10.88^{* * * *}$ & $4.67^{* * *}$ & $10.00^{*}$ \\
\hline & \multirow{9}{*}{ 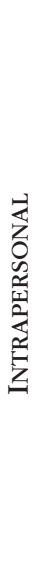 } & UNA VIDA EXCITANTE (activa, estimulante) & $18.83^{* * * * *}$ & $5.42 * *$ & $37.88^{* * * *}$ \\
\hline & & $\begin{array}{l}\text { SENTIDO DE REALIZACIÓN (lograr } \\
\text { realizarse) }\end{array}$ & 0.21 & 3.68 & 3.43 \\
\hline & & LIBERTAD (independencia, elección libre) & 0.53 & 3.01 & 0.09 \\
\hline & & FELICIDAD (satisfacción) & 0.03 & 0.00 & $26.50 * * * *$ \\
\hline & & $\begin{array}{l}\text { ARMONÍA INTERIOR (sin conflictos } \\
\text { internOS) }\end{array}$ & 0.99 & $6.76^{* * * *}$ & $4.00^{*}$ \\
\hline & & $\begin{array}{l}\text { PLACER (una vida agradable } \\
\text { y placentera) }\end{array}$ & $5.07^{* * *}$ & 2.00 & $22.81^{* * * * *}$ \\
\hline & & SALVACIÓN (una vida eterna) & $5.77^{*}$ & 0.19 & $8.17^{*}$ \\
\hline & & AMOR PROPIO (autoestima) & 0.35 & $10.10^{* * * * *}$ & $10.18^{*}$ \\
\hline & & $\begin{array}{l}\text { SABIDURÍA (buena comprensión } \\
\text { de la vida) }\end{array}$ & 2.03 & 2.79 & $13.58^{* * * * *}$ \\
\hline
\end{tabular}

Como ya habíamos apuntado, una vida excitante es más valorada por la cohorte de jóvenes. Con este análisis hallamos también que es más estimada por 
mayores con estudios $(\mathrm{Me}=12)$ que por el grupo sin estudios $(\mathrm{Me}=15)$. En cuanto a las diferencias intersexo, observamos una mayor valoración por parte de los varones $(\mathrm{Me}=12)$ que en las mujeres de la muestra $(\mathrm{Me}=14)$. Yagüe et al. (2013) encuentran las mismas diferencias significativas en su investigación.

La madurez en el amor, un valor de prioridad global media, adquiere más importancia dentro del grupo de jóvenes y personas con estudios, y también es más priorizado por el sexo masculino que por el femenino. Las diferencias más significativas se encuentran en relación a la cohorte y al nivel de estudios, con una diferencia de 3 posiciones en relación a sus medianas tanto a favor de la cohorte de jóvenes, como de las personas con estudios.

La amistad verdadera es, en general, un valor de prioridad media-alta y para la cohorte de jóvenes y el sexo femenino se convierte en un valor especialmente importante, situándose en ambos grupos en una mediana de 7 . Yagüe et al. encuentran, asimismo, que este valor es más estimado por jóvenes; sin embargo, en su estudio, es algo más valorado por hombres que por mujeres.

Si nos centramos en las diferencias significativas intercohorte, encontramos entre los valores finalistas -tanto interpersonales como intrapersonales- un 55.55\% de diferencias significativas entre jóvenes y mayores. Se destacan los valores un mundo en paz, seguridad nacional, seguridad familiar y sabiduria, priorizados más por la cohorte de más edad, con medianas de 4, 12, 4 y 8 respectivamente. En cambio, en estos mismos valores, los jóvenes obtuvieron medianas de 11, 16, 7 y 10. Esta menor valoración de los jóvenes implicaría, asimismo, un mayor nivel de postmaterialismo, reafirmado por el dato de que los valores hedonistas de felicidad y placer son más apreciados por el grupo de jóvenes, con medianas de 2 y 9, mientras que para los adultos y mayores las medianas se situaron en 5 y 12 respectivamente.

Entre las diferencias por nivel de estudios observamos que en los valores finalistas denominados interpersonales se producen diferencias estadísticamente significativas en más de la mitad de los valores analizados. Las mayores discrepancias se observan en los valores: una vida confortable, un mundo en paz y seguridad nacional que son más valorados por el grupo de personas sin estudios.

En relación a los valores finalistas intrapersonales, se observa un 33.33\% de diferencias según la variable nivel de estudios, obteniendo el valor placer la discrepancia más significativa siendo más valorado por las personas mayores con estudios.

\section{CONCLUSIONES, LIMITACIONES Y PROSPECTIVA}

Desde que en la década de los setenta se formulara la hipótesis del cambio de valores del modernismo al postmodernismo no han dejado de aumentar los trabajos sobre el análisis de los valores, así como sus significados e implicaciones. En la actualidad, la crisis e inestabilidad económica y social que se vive en las sociedades occidentales está generando un nuevo cambio de prioridades y de valores, que 
sitúa al postmodernismo en el punto de mira, en cuanto a su validez para afrontar ciertas situaciones de inseguridad, que retrotraerían hacia planteamientos en busca de la estabilidad y la protección, más propios del modernismo (Todd, 2009).

Por un lado, las críticas contra el cambio de valores que propondría la condición postmoderna (Bauman, 2005), junto a la necesidad de priorizar valores tradicionales, han llevado a un statu quo que en parte ha contribuido a que el proyecto de vida postmodernista no sea ya una finalidad a alcanzar (Martínez, Esteban y Buxarrais, 2011). En este sentido coincidimos con Brenneman y Margonis (2012) en que debemos movernos más allá de proyectos educativos inspirados en las promesas del modernismo o del postmodernismo, porque lo más relevante sería enseñar a los educandos a estimar y aplicar valores sin una etiqueta asociada al pasado o al presente, sino a través de su validez intrínseca para reflexionar y afrontar las diferentes condiciones vitales. Como ya hemos indicado, los valores pueden situarse en un continuo y, precisamente, en nuestro estudio fueron los valores situados en el medio del continuo materialismo-postmaterialismo (honestidad, responsabilidad o tolerancia) los que fueron estimados como prioritarios de manera significativamente similar por ambos grupos de edad.

Por otro lado, además de la importancia que ejercen las contingencias sociales, a nivel macro, como es en este caso la vivencia de la crisis económica, no podemos olvidar el efecto que tiene la condición evolutiva para dirigir las preferencias en función de la edad y las experiencias vitales a las que se puede aspirar (valores finalistas). En esta idea se fundamenta la denominada brecha intergeneracional entre los valores que son priorizados por jóvenes y mayores. En este trabajo hemos partido principalmente de las hipótesis de Inglehart, revelando una mirada en cierto modo distinta respecto a la brecha intergeneracional en el ámbito de los valores. En nuestra muestra se observa mayor coincidencia entre jóvenes y mayores, al igual que en otras investigaciones recientes (Alonso et al., 2013; Yagüe et al., 2013), en lo que se refiere a valores básicos instrumentales. Nos planteamos que este acercamiento entre cohortes podría estar influido por los efectos de la crisis económica mundial. Siguiendo las hipótesis originales de escasez y socialización de Inglehart, podría suponerse que, debido a la crisis, muchos jóvenes estarían viviendo en su proceso de socialización un periodo de escasez material, similar al que vivieron personas de generaciones anteriores, socializadas en los 50 o décadas anteriores, lo que les retraería a valores algo más materialistas. Sin embargo, nuestros datos muestran también, de manera contundente, y coincidiendo con otras investigaciones (Gil Roales-Nieto y Segura, 2010; Alonso et al., 2013; Ryan, Schruder y Robinson, 2013), un mayor índice de postmodernismo en los jóvenes. Por una parte se observa en ellos mayor dispersión y diversidad respecto a los valores más priorizados, lo que correspondería a una mayor pluralidad y relativismo, características propias de la postmodernidad. Por otra, los jóvenes eligen prioritariamente valores finalistas más intrapersonales e individualistas como placer o vida excitante, mientras que los mayores eligen valores finalistas más materialistas e interpersonales como un mundo en paz, vida confortable, seguridad nacional o seguridad 
familiar. Es posible, no obstante, que como venimos comentando algunas de estas preferencias puedan deberse no solo a los cambios macrosociales, sino fundamentalmente al momento del ciclo vital que atraviesan y que dirige decisivamente sus aspiraciones.

Un dato interesante, coincidente con las ideas originales de Inglehart, es que las diferencias intergeneracionales aumentan cuando comparamos al grupo de jóvenes con el grupo de mayores sin estudios. Un mayor nivel de estudios entre las personas mayores parece acercarles más al postmodernismo y a los jóvenes en cuanto a estimación de valores se refiere. Asimismo, en lo que se refiere a las diferencias intersexo, las más significativas se producen al priorizar más las mujeres valores como la amistad verdadera, la armonía interior, el amor propio o la limpieza, mientras que los varones colocan en niveles más altos valores como la vida excitante, la madurez en el amor o el reconocimiento social. Curiosamente las mujeres de nuestro estudio han priorizado el valor ambición más que los hombres, lo que parece ir en contra de los estereotipos de género transmitidos a niños y niñas en los procesos de socialización. Es esta variable sexo, junto a la que refiere al nivel de estudios, las que pensamos que, en próximas investigaciones, podrían estudiarse de forma más exhaustiva.

En definitiva, si el postmodernismo, con sus ventajas e inconvenientes, se encuentra girando hacia valores más materialistas por efecto de los problemas socioeconómicos, es preciso que los educadores seamos conscientes de ello, con el fin de abordar el complejo proceso educativo que exige la construcción de valores. Si los educandos han vivido un contexto socioeconómico de inseguridad social y económica antes de la madurez, tendríamos que prever hasta qué punto esto se reflejará en su priorización de valores, y con ello en su carácter y conducta moral.

Para concretar un poco más la propuesta educativa que derivaría de nuestro estudio, en primer lugar, consideramos relevante tener claras las prioridades axiológicas de los jóvenes. En este sentido resulta imprescindible mejorar la formación de los docentes, con el fin de que aprendan a explorar los valores que estiman sus alumnos, para que una vez explícitas sus preferencias puedan desarrollar metodologías eficaces que les lleven a reflexionar sobre los valores que son útiles y necesarios en contextos tan diversos y cambiantes como el que vivimos actualmente. Explorar qué estiman y qué sería necesario que aprendieran a estimar, teniendo en cuenta factores micro y macro, podría dar como resultado una unificación de criterios y esfuerzos pedagógicos y didácticos, con claros beneficios en la modificación y actualización de programaciones, planes de acción tutorial y programas de formación del profesorado. Porque, a pesar de la falta de consenso sobre cuáles son los verdaderos valores a trabajar y potenciar en las aulas, la educación en valores sigue siendo crucial, ya sea enseñando matemáticas, literatura o inglés, siendo insuficiente con una asignatura, llámese Valores Éticos, Valores Culturales y Sociales, Educación para la Ciudadanía o Educación en Valores. Los docentes necesitan aprehender métodos cooperativos en lugar de competitivos, dialógicos en lugar de impositivos, que faciliten 
ponerse en el lugar de los demás y desarrollen empatía, que enseñen a escuchar, contrastar opiniones y conseguir acuerdos, que conduzcan a aprender a respetar y valorar la diversidad, a ejercitar la solidaridad.

En segundo lugar, consideramos necesario que el currículum escolar contemple de forma más amplia y precisa el desarrollo de valores interpersonales en los jóvenes, quienes, como hemos visto, tienden a priorizar los valores individualistas. Para lograr la construcción de valores prosociales (interpersonales) se necesita un enfoque transversal y continuo, es decir; hay que tenerlo en cuenta en todas y cada una de las materias del currículum, de manera coordinada en las diferentes etapas. Así lo contempla en este momento la actual ley de educación (LOMCE), sin embargo, habrá que estar muy atentos a la forma de implementarlo, puesto que se requieren determinadas condiciones básicas, como una adecuada formación del profesorado, o contextos (por ejemplo, de aprendizaje-servicio) que provean de oportunidades para llevar a la práctica los valores aprendidos.

En tercer lugar, en nuestro contexto democrático sigue siendo imprescindible la actitud dialógica y el consenso para educar en valores. Como ya algunos autores han subrayado (Martínez, Esteban y Buxarrais, 2011), son fundamentales la reflexión pedagógica compartida y sobre todo el diálogo, con el fin de llegar a acuerdos y conseguir pautas y criterios comunes acerca de los principios morales y los valores en sociedades tan cambiantes. El hecho de que en nuestro estudio las posibles diferencias intergeneracionales son más marcadas en los valores finalistas, y menos en los instrumentales, más básicos, como la tolerancia, la solidaridad o la concordia, pone de manifiesto que ciertos valores pueden ser más necesarios, especialmente en los contextos socioculturales contemporáneos. Respecto a los estudiantes adultos-mayores que, como hemos visto, presentan un menor índice de postmodernismo, sería interesante trabajar educativamente para facilitar el análisis y la crítica reflexiva de las «verdades» absolutas y la autoridad marcada; la desconfianza hacia el pensamiento único y la apreciación de la pluralidad de opiniones y valores, utilizando asimismo metodologías dialógicas y activas.

A nivel metodológico, algunos autores han criticado la estrategia de ordenar los valores por orden de preferencia en escalas como la utilizada en este estudio (RSV de Rokeach), por su posible efecto distorsionador de resultados (Tranter y Western, 2004). Según las recientes revisiones, todos los métodos existentes para evaluar valores, plantean ventajas e inconvenientes, y la Rvs no es una excepción. Consideramos que una limitación de la prueba es la obligación de relegar algunos valores a los últimos lugares, lo cual no significa que no sean estimados, sino que la prueba no permite otorgarles la importancia real que la persona les atribuye. Este inconveniente podría ser solventado, como ya han sugerido Lila, Musitu y Buelga (2000), si, en un segundo momento, se diera la oportunidad a los/las participantes de asignar una valoración individual para cada uno de los valores. Ello permitiría al sujeto la posibilidad de otorgar, por ejemplo, la misma importancia a dos o más valores, lo que no es posible en la versión actual del instrumento. 
Para finalizar, y de cara a ulteriores investigaciones, consideramos que es preciso profundizar en los posibles efectos de la crisis socioeconómica sobre los valores, para ratificar si realmente existe un mayor acercamiento intergeneracional y un cierto retroceso desde valores postmaterialistas hacia valores más materialistas, especialmente en los jóvenes. En este sentido se necesitan más investigaciones longitudinales, que analicen la priorización axiológica en individuos cuya infancia y primera juventud haya transcurrido en el periodo más intenso de la crisis, y qué efectos tiene en un futuro lejano dicha experiencia vivida para la priorización de valores.

\section{REFERENCIAS BIBLIOGRÁFICAS}

Alonso, Y.; Jiménez-López, F.; García-Vargas, G. y Gil Roales-Nieto, J. (2013) Value Change and Post-modernism: A Preliminary Study of a German Sample. International Journal of Psychology \& Psychological Therapy, 13 (3), 277-287.

Bardi, A.; Buchanan, K. E.; Goodwin, R.; Slabu, L. y Robinson, M. (2014) Value stability and change during self-chosen life transitions: Self-selection versus socialization effects. Journal of personality and social psychology, 106 (1), 131-147.

BARDI, A. y Goodwin, R. (2011) The dual route to value change: Individual processes and cultural moderators. Journal of Cross-Cultural Psychology, 42 (2), 271-287.

BARRIO, J. M. (2008) Sobre la llamada educación posmoderna. Revista Española de Pedagogía, 241, 527-540.

Bauman, Z. (2001) Los retos de la educación en la modernidad líquida. Barcelona, Gedisa.

Bauman, Z. (2005) Ética posmoderna. México, Siglo Xxi Editores.

BAuman, Z. (2007) Los retos de la educación en la modernidad líquida. Barcelona, Gedisa.

BECK, U. (1998) La sociedad del riesgo. Hacia una nueva modernidad. Barcelona, Paidós.

Beltrán Guzmán, F. J.; Torres Fermán, I.; Beltrán Torres, A. y García Díaz, F. (2005) Un estudio comparativo sobre valores éticos en estudiantes universitarios. Enseñanza e Investigación en Psicología, 10 (2), 397-415.

Bericat, E. (2003) Fragmentos de la realidad social posmoderna. Revista Española de Investigaciones Sociológicas, 102, 9-46.

Boudon, R. y Bourricaud, F. (1990a) A Critical Dictionary of Sociology. Chicago, University of Chicago.

Boudon, R. y Bourricaud, F. (1990b) Diccionario crítico de la sociología. Madrid, Edicar.

Brenneman, M. y Margonis, F. (2012) Degrees of disenchantment: A review essay. Educational Theory, 62 (2), 225-247.

Buss, D. M. y Hawley, P. (2011) The evolution of personality and individual differences. Nueva York, Oxford University Press.

Cheng, A. S. y Fleischmann, K. R. (2010) Developing a Meta-Inventory of Human Values. ASIST, 22-27. Pittsburg, P. A. USA.

Colom, T. y Melich, J. C. (1993) Postmodernidad y educación. La teoría de Toffler y la práctica de la C. M. U. Teoría de la Educación. Revista Interuniversitaria, V, 97-110.

Damme, D. V. y KarkKainen, K. (2011) OeCD Education today Crisis Survey 2010: The Impact of the Economic Recession and Fiscal Crisis on Education in OECD Countries. OECD Education Working Papers, 56, OECD Publishing. 
M. J. HERNÁNDEZ SERRANO, G. PARRA NIETO Y M. D. PÉREZ GRANDE

PRIORIZACIÓN DE VALORES EN ESTUDIANTES JÓVENES Y MAYORES EN EL CONTEXTO DE LA CRISIS ECONÓMICA

Daset Carreto, L.; López Martirena, A. y Suero Girardi, M. (2007) Valores Humanos Básicos en un grupo de jóvenes: avance de un estudio exploratorio. Psicodebate (7), 41-54.

Díez Nicolás, J. (2011) ¿Regreso a los valores materialistas? El dilema entre seguridad y libertad en los países desarrollados. RES, 15, 9-46.

EDWARDS, R. (2006) All quiet on the postmodern front? Studies in Philosophy and Education, 25 (4), 273-278.

Elzo, J. y CastiÑeIRA, A. (2011) Valors tous en temps durs: la societat catalana a l'Enquesta europea de valors de 2009. Barcelona, Barcino.

Fernández Alonso, M. (2010) Valores y creencias en el proceso de socialización. Papers, 95/4, 1031-1051.

Fullat, O. (2003) Heidegger o los valores rasos de la educación postmoderna. Revista Española de Pedagogía, 225, 209-222.

García Alandete, J. y Pérez Delgado, E. (2005) Razonamiento moral y valores: estudio de sus relaciones en un grupo de universitarios españoles. Revista Latinoamericana de Psicología, 37, 131-148.

García del Dujo, Á. y Mínguez Vallejos, R. (2011) Los límites de los valores cívicos: cuestiones y propuestas pedagógicas. Educación Xx1, 14 (2), 263-285.

Gervilla, E. (2002) La tiranía de la belleza, un problema educativo hoy. Teoría de la Educación. Revista Interuniversitaria, 14, 185-206.

Gervilla, E. (2011) Educar en la Postmodernidad. Madrid, Dykinson.

Gil Roales-Nieto, J. (2009) Cambio social y cambio personal. Estudio preliminar en una muestra intergeneracional. International Journal of Psychology and Psychological Therapy, 9, 395-420.

Gil Roales-Nieto, J. y Segura, A. (2010) Intergenerational Differences in Materialism and Postmaterialism Values in a Spanish Sample. International Journal of Psychology and Psychological Therapy, 10 (3), 499-512.

GuR-ZE'Ev, I. (2007) Beyond the Modern-Postmodern Struggle in Education: Toward CounterEducation and Enduring Improvisation. Rotterdam, Netherlands, Sense Publishers.

Hoffmann, J. G. (2009) The multidimensional structure and function of human values. University of Southern California. Doctoral Dissertation.

INGLEHART, R. (1977) The silent revolution: Changing values and political styles among western publics. Princeton, Princeton University Press.

Inglehart, R. (1981) Post-materialism in an environment of insecurity. American Political Science Review, 75 (4), 124-137.

Inglehart, R. (1990) Culture shift in advanced industrial societies. Princeton, Princeton University Press.

INGLEHART, R. (1991) El cambio cultural en las sociedades industriales avanzadas. Madrid, Siglo XXI.

Inglehart, R. (1995) Measuring postmaterialism. American Political Science Review, 93 (3), 144-157.

Inglehart, R. (1997a) Modernization and Postmodernization: Cultural, Economic and Political Change in 43 Societies. Princeton, Princeton University Press.

IngleHART, R. (1997b) The trend towards postmaterialist values continues, en ClaRK, N. y Rempel, M. (eds.) Citizen Politics in Post-industrial societies. Boulder and Oxford, Westview Press, 162-168.

IngleHART, R. (2000) Globalization and Postmodern Values. Washington Quarterly, 23 (1), 215-228. 
Inglehart, R. y Abramson, P. R. (1994) Economic Security and Value Change. American Political Science Review, 88, 336-354.

Inglehart, R. y Klingemann, H. D. (1996) Dimensions of value change. Theoretical and methodological reflection on the occasion of new criticism. Politische Vierteljahresschrtift, 37, 319-340.

Inglehart, R. y Welzel, C. (2005) Modernization, Cultural Change, and Democracy: The Human Development Sequence. Cambridge, Cambridge University Press.

Inglehart, R. y Welzel, C. (2006) Emancipative values and democracy: Response to Hadenius and Teorell. Studies in Comparative International Development, 41 (3), 74-94.

JiMÉNEZ-LópeZ, F. R. (2011) Análisis del cambio intergeneracional de valores en estudiantes y profesionales de Enfermería. Tesis doctoral. Universidad de Almería, España.

Kemmis, S. (2000) Aspiraciones emancipadoras en la era posmoderna. Kikiriki, 55/56, 14-34.

Klein, M.; DÜlmer, H.; OHR, D.; Quandt, M. y Rosar, U. (2004) Response sets in the measurement of values: A comparison of rating and ranking procedures. International Journal of public Opinion Research, 16, 474-483.

Klein, M. y PÖTsChKE, M. (2000) Gibt es einen Wertewandel zum «reinen» Postmaterialismus? Eine Zeitreihenanalyse der Wertorientierungen der westdeutschen Bevölkerung zwischen 1970 und 1997. Zeitschrift für Soziologie, 29, 202-216.

KOHLBERG, L. (1976) Moral stages and socialization. The cognitive-developmental approach, en Lickona, T. (ed.) Moral development and behavior. New York, Holt-RinehartWinston, 31-53.

LAUDO, X. y PRATS, E. (2013) El lugar de la autonomía en la pedagogía y la educación postmodernas. Teoría de la Educación: Educación y Cultura en la Sociedad de la Información, 14 (1), 248-262.

Lila, M.; Musitu, G. y Buelga, S. (2000) Adolescentes colombianos y españoles: Diferencias, similitudes y relaciones entre la socialización familiar, la autoestima y los valores. Revista Latinoamericana de Psicología, 32, 301-319.

Lipovetsky, G. (1986) La era del vacío. Ensayos sobre el individualismo contemporáneo. Barcelona, Anagrama.

Lipovetsky, G. y Charles, S. (2006) Los tiempos hipermodernos. Barcelona, Anagrama.

Liubiniene, V. (2003) Value orientations in Estonia and Lithuanian Society: sociological analysis from the gender and age perspective. Socialiniai mokslai, 3 (40), 45-53.

Martí, M. y Palma, J. (2010) Jerarquización y preferencia de valores en los estudiantes de secundaria. Revista Española de Orientación y Psicopedagogía, 21 (3), 603-616.

Martínez, M.; Esteban, F. y Buxarrais, M. R. (2011) Escuela, profesorado y valores. Revista de Educación (n. ${ }^{\circ}$ extraordinario), 95-113.

Mayos, G. (2011) La sociedad de la incultura, ¿Cara oculta de la sociedad del conocimiento?, en Mayos, G. y Brey, A. (eds.) La sociedad de la ignorancia. Barcelona, Península, $167-227$.

Myyry, L.; Juujärvi, S. y Pesso, K. (2013) Change in values and moral reasoning during higher education. European Journal of Developmental Psychology, 10 (2), 269-284.

Norris, P. e Inglehart, R. (2011) Sacred and secular: Religion and politics worldwide. Cambrige, Cambrige University Press.

Ovadia, S. (2004) Ratings and rankings: reconsidering the structure of values and their measurement. Internacional Journal Social Research Methodology, 7 (5), 403-414. 
M. J. HERNÁNDEZ SERRANO, G. PARRA NIETO Y M. D. PÉREZ GRANDE

PRIORIZACIÓN DE VALORES EN ESTUDIANTES JÓVENES Y MAYORES EN EL CONTEXTO DE LA CRISIS ECONÓMICA

Penas Castro, S. (2008) Aproximación a los valores y estilos de vida de los jóvenes de 13 y 14 años en la provincia de A Coruña. Universidad de Santiago de Compostela. Tesis doctoral publicada.

Perrenoud, P. (2001) La formación de los docentes en el siglo XXI. Revista de Tecnología Educativa, 14 (3), 503-523.

Piaget, J. (1932) El juicio moral del niño. Madrid, Francisco Beltrán Editor.

Reimer, B. (1988) No Values-New Values? Youth and Postmaterialism. Scandinavian Political Studies, 11 (4), 347-360.

Rest, J. R.; Narvaez, D.; Thomas, S. J. y Bebeau, M. J. (2000) A Neo-Kohlbergian Approach to Morality Research. Journal of Moral Education, 29 (4), 381-395.

ReYnolds, B. K. (1984) A cross-cultural study of values of Germans and Americans. Internacional Journal of Intercultural Relations, 8, 269-278.

Rokeach, M. (1968) Beliefs, Attitudes and Values: A Theory of Organization and Change. San Francisco, Jossey-Bass Publishers.

Rokeach, M. (1973) The Nature of Human Values. New York, The Free Press.

Rokeach, M. (1979) Some unresolved issues in theories of beliefs, attitudes, and values. Nebraska Symposium on Motivation, 27, 261-304.

Rumbo Arcas, B. (2013) Tensiones y dilemas en la educación de personas adultas. Teoría de la Educación: Educación y Cultura en la Sociedad de la Información, 14 (2), 178-191.

RyAn, T. G.; SCHRUder, C. R. y RoBinson, S. (2013) Concurrent pre-service teachers: An analysis of values. Issues in Educational Research, 23 (3), 394-414.

SANTOS GuerRa, M. Á. (2010) Una pretensión problemática: educar para los valores y preparar para la vida. Revista de Educación, 351, 23-47.

SchwarTz, H. S. y SAGIE, G. (2000) Value consensus and importance: a cross-national study. Journal of Cross-cultural Psychology, 31 (4), 465-497.

SENNETT, R. (2000) La corrosión del carácter. Las consecuencias del trabajo en el nuevo capitalismo. Barcelona, Anagrama.

TodD, S. (2009) Toward an imperfect education: Facing humanity, rethinking cosmopolitanism. Colorado: Paradigm Publishers.

TorCaL, M. (1989) La dimensión materialista/postmaterialista en España: Las variables del cambio cultural. Reis: Revista Española de Investigaciones Sociológicas, 47, 227-254.

Tranter, B. y Western, M. (2004) Question order effects in Inglehart's postmaterial index. Australasian Political Studies Association Conference, University of Adelaide, 1-27.

Tranter, B. y Western, M. (2010) Overstating Value Change: Question Ordering in the Postmaterial Values Index. European Sociological Review, 26 (5), 571-583.

Tulviste, T. y Tамm, A. (2014) Brief report: Value priorities of early adolescents. Journal of Adolescence, 37 (5), 525-529.

Wain, K. (2008) The future of education... and its philosophy. Studies in Philosophy and Education, 27 (2-3), 103-114.

Yagüe López, L.; Sánchez Rodríguez, A. I.; De Miguel Negredo, A. y Gómez Becerra, I. (2013) Sexo y cohorte como fuentes de diferenciación en valores en la sociedad postmoderna. International Journal of Psychology and Psychological Therapy, 13 (2), 243-253. 